\title{
Observation of cosmic ray anisotropy with GRAPES-3 Experiment
}

\author{
P.K. Mohanty ${ }^{* 1,2}$, S. Ahmad ${ }^{1,4}$, M. Chakraborty ${ }^{1,2}$, A. Chandra ${ }^{1,4}$, S.R. Dugad ${ }^{1,2}$, \\ S.K. Gupta ${ }^{1,2}$, B. Hariharan ${ }^{1,2}$ Y. Hayashi ${ }^{1,5}$, P. Jagadeesan ${ }^{1,2}$, A. Jain ${ }^{1,2}$, P. Jain ${ }^{1,6}$, \\ V.B. Jhansi ${ }^{1,2}$, S. Kawakami ${ }^{1,5}$, H. Kojima ${ }^{1,7}$, S. Mahapatra ${ }^{1,8}$, S.D. Morris ${ }^{1,2}$, \\ P.K. Nayak ${ }^{1,2}$, A. Oshima ${ }^{1,7}$, D. Pattanaik ${ }^{1,2}$, P.S. Rakshe ${ }^{1,2}$, K. Ramesh ${ }^{1,2}$, \\ B.S. Rao ${ }^{1,2}$, L.V. Reddy ${ }^{1,2}$, S. Shibata ${ }^{1,7}$, F. Warsi ${ }^{1,6}$, M. Zuberi ${ }^{1,2}$ \\ ${ }^{1}$ The GRAPES-3 Experiment, Cosmic Ray Laboratory, Raj Bhavan, Ooty 643001, India \\ ${ }^{2}$ Tata Institute of Fundamental Research, Mumbai 400005, India \\ ${ }^{4}$ Aligarh Muslim University, Aligarh 202002, India \\ ${ }^{5}$ Graduate School of Science, Osaka City University, Osaka 558-8585, Japan \\ ${ }^{6}$ Indian Institute of Technology Kanpur, Kanpur 208016, India \\ ${ }^{7}$ College of Engineering, Chubu University, Kasugai, Aichi 487-8501, Japan \\ ${ }^{8}$ Utkal University, Bhubaneshwar 751004, India \\ E-Mail: pkmetifr.res.in
}

\begin{abstract}
Large scale anisotropy of cosmic rays in the TeV-PeV energy region has been reported by several experiments located in the Northern Hemisphere as well as by the IceCube, and its surface array IceTop at the South Pole. The GRAPES-3 experiment in Ooty, India is designed to observe cosmic rays in the energy range from a few $\mathrm{TeV}$ to over $10 \mathrm{PeV}$. Its near-equatorial location $\left(11.4^{\circ} \mathrm{N}\right)$ provides a unique advantage of its observations on cosmic ray anisotropy, overlapping with experiments in both the hemispheres. The GRAPES-3 consists of a dense array of 400 plastic scintillator detectors spread over an area of $25000 \mathrm{~m}^{2}$. It also contains a large area (560 $\mathrm{m}^{2}$ ) tracking muon detector which provides an excellent capability to discriminate $\gamma$-rays against cosmic rays. It has recorded more than 10 billion showers since its operation began in 2000 . Measurement of cosmic ray anisotropy with a subset of this data is presented here.
\end{abstract}

36th International Cosmic Ray Conference -ICRC2019-

July 24th - August 1st, 2019

Madison, WI, U.S.A.

${ }^{*}$ Speaker. 


\section{Introduction}

Understanding the origin of cosmic rays (CRs) is a fundamental problem in astrophysics which has remained unresolved even after their discovery more than a century ago. This could be attributed to the fact that the CRs are charged particles because of which their directions are randomized by the magnetic field in the interstellar medium through which they propagate. This leads to the arrival directions of CRs to be sotropic when observed on Earth making it impossible to trace their sources. The CRs below $10^{17} \mathrm{eV}$ are considered to be originated in our Galaxy. They are mostly believed to be produced and accelerated by shocks in supernova remnants [1]. The galactic magnetic field is $\sim 3 \mu \mathrm{G}$ contains a regular and a turbulent component. The latter is believed to have a scale-size much larger than the Larmor radii for CRs below $10^{17} \mathrm{eV}$. However, during the past one decade, several ground based air shower arrays have observed a small anisotropy in the arrival directions of CRs at the level of $10^{-4}$ to $10^{-3}$ in the energy range of $\mathrm{TeV}-\mathrm{PeV}$. An excess in CR flux is observed in a region $40^{\circ}-90^{\circ}$ in right ascension and a deficit in a region $150^{\circ}-240^{\circ}$ in right ascension [2]. Thanks to large statistics collected by these experiments with duty cycle nearly $100 \%$ and a field of view covering the full sky making it possible to detect this exceedingly effect. The cause of anisotropy may be explained by the presence of an ordered magnetic field in our local environment with a strength at the level of $3 \mu \mathrm{G}$ which induces a circular motion of CRs around magnetic field lines with a Larmor radius $\mathrm{r}_{\mathrm{L}} \simeq 0.4 \mathrm{E}_{\mathrm{PeV}} /\left(\mathrm{ZB}_{3 \mu G}\right) \mathrm{pc}$. This length scale is much smaller than the typical scattering length for $\mathrm{CRs}$ in the $\mathrm{TeV}-\mathrm{PeV}$ energies predicted by isotropic diffusion models which may indicate a strong anisotropic diffusion in our local environment [3]. Thus, the study of the CR anisotropy may provide important information on their source distribution as well as the structure of the magnetic field.

Several experiments have measured the large scale CR anisotropy in the $\mathrm{TeV}-\mathrm{PeV}$ energies including Tibet AS $\gamma$ [4], ARGO-YBJ [5], Milagro [6], HAWC [7], EAS-TOP [8], IceCube/IceTop $[9,10]$. Each of these experiments is sensitive in a different energy range of the CR spectrum. A recent compilation of CR anisotropy results shows that the amplitude is energy dependent and phase flip occurs around $100 \mathrm{TeV}$ [7]. Two dimensional mapping of CR anisotropy in right ascension and declination coordinates has been successfully carried out by several of these experiments due to the high statistics data collected by them. The IceCube and IceTop being located at the South pole are sensitive to CR anisotropy in the southern hemisphere while the rest of the experiments are located in the northern hemisphere. The fortuitous location of GRAPES-3 air shower array at Ooty in India close to the equator $\left(11.4^{\circ} \mathrm{N}\right)$ provides the unique advantage of observing CR anisotropy with overlapping fields of view of detectors with both the hemispheres. The GRAPES-3 also could provide a good overlap of CR anisotropy measurement in energy as it is sensitive from a few $\mathrm{TeV}$ to over PeV.

\section{The GRAPES-3 experiment}

The GRAPES-3 experiment consists a dense array of scintillator detectors with a coverage area of $25,000 \mathrm{~m}^{2}$. It is currently operating with 400 scintillator detectors of $1 \mathrm{~m}^{2}$ area each with $8 \mathrm{~m}$ inter-detector separation arranged in a hexagonal configuration [11, 12]. Each scintillator detector is designed to measure densities and arrival times of shower particles to obtain energy and incident 
direction of primary CRs. It began operating in year 2000 with a modest array of 217 plastic scintillator detectors of a traditional type [11]. It was subsequently upgraded and expanded with a vastly superior design based on in-house produced plastic scintillator and wavelength shifting fiber obtained from Kuraray, Japan [12]. The trigger rate of the array is currently $35 \mathrm{~Hz}$ which is three times higher compared to the rate prior to the upgrade. The PMT signal from each detector is digitized using a charge integrating CAEN ADC and the relative arrival times are digitized using an in-house developed 32-channel TDC module namely HPTDC based on an application specific integrated circuit designed and developed by the micro-electronics group at CERN [13]. The other major component of the GRAPES-3 experiment is a large area $\left(560 \mathrm{~m}^{2}\right)$ tracking muon detector that consists of 16 separate modules of $35 \mathrm{~m}^{2}$ area each [14]. Each module consists of 4 layers of proportional counters (PRC) made of $6 \mathrm{~m}$ long and $0.1 \mathrm{~m} \times 0.1 \mathrm{~m}$ square cross section steel tubes to track individual muons that survive after passing through a concrete absorber of $550 \mathrm{gm}$ $\mathrm{cm}^{-2}$ which filters out the electromagnetic components and sets a threshold of $1 \mathrm{GeV}$ for vertically incident muons. The muon detector provides an excellent discrimination of $\gamma$-rays against charged CRs enabling the study of multi $\mathrm{TeV} \gamma$-ray sources. In addtion, the muon component provides a sensitive measurement of CR composition [15]. The GRAPES-3 has recorded over 10 billion shower events since its operation began in 2000. A recent detail simulation study has shown that $50 \%$ of showers recorded by the array are below $10 \mathrm{TeV}$.

\section{Data analysis, results and discussion}

In this report, we present the analysis of data carried out for the period 2000-2006 comprising about two billion showers. The shower direction (zenith angle $\theta$ and azimuth angle $\phi$ ) was determined using a plane fit to the relative arrival times of shower front at various detectors after correcting for the shower front curvature with a slope of $215 \mathrm{ps} \mathrm{m}^{-1}$. Details of the direction reconstruction and angular resolution study can be found elsewhere [16]. The study has found that the angular resolution of the GRAPES- 3 array is $1.4^{\circ}$ at $10 \mathrm{TeV}$ which improves to $0.4^{\circ}$ at $80 \mathrm{TeV}$. The angular resolution obtained using three different methods namely even-odd, left-right and Moonshadow have provided consistent results. Various other shower parameters such as core location $\left(\mathrm{X}_{c}, \mathrm{Y}_{c}\right)$, age 's' and size ' $\mathrm{N}_{\mathrm{e}}$ ' were obtained by fitting $\mathrm{NKG}$ function to the lateral distribution of particle densities through a minimization algorithm using MINUIT which is discussed elsewhere [15].

The arrival direction of each shower $(\theta, \phi)$ was converted into equatorial coordinates, right ascension $(\alpha)$ and declination $(\delta)$. Then this data was binned into cells of size $3^{\circ} \times 3^{\circ}$ in $\alpha$ and $\delta$ where $\alpha$ varies from $0^{\circ}$ to $360^{\circ}$ and $\delta$ varies from $-90^{\circ}$ to $90^{\circ}$. Events in each cell was normalized with the average value for the corresponding declination band. The anisotropy was calculated by subtracting a dummy cosmic ray distribution from the real cosmic ray distribution where the dummy distribution was produced using the real data by shuffling event time. The statistical significance value for each bin was calculated using Li Ma formula [17]. A 2-D sky map of the significance without applying any smoothing is shown in Fig.1. The significance of same data in three different declination bands for $\delta$ between $+40^{\circ}$ and $-20^{\circ}$ is shown in Fig.2.

The significance map in Fig. 1 indicates an excess in a broad region of $\alpha=50^{\circ}-130^{\circ}$ and a deficit in $\alpha=150^{\circ}-230^{\circ}$. The anisotropy is clearly visible over $\delta=+40^{\circ}$ in northern hemisphere and $\delta=-30^{\circ}$ 


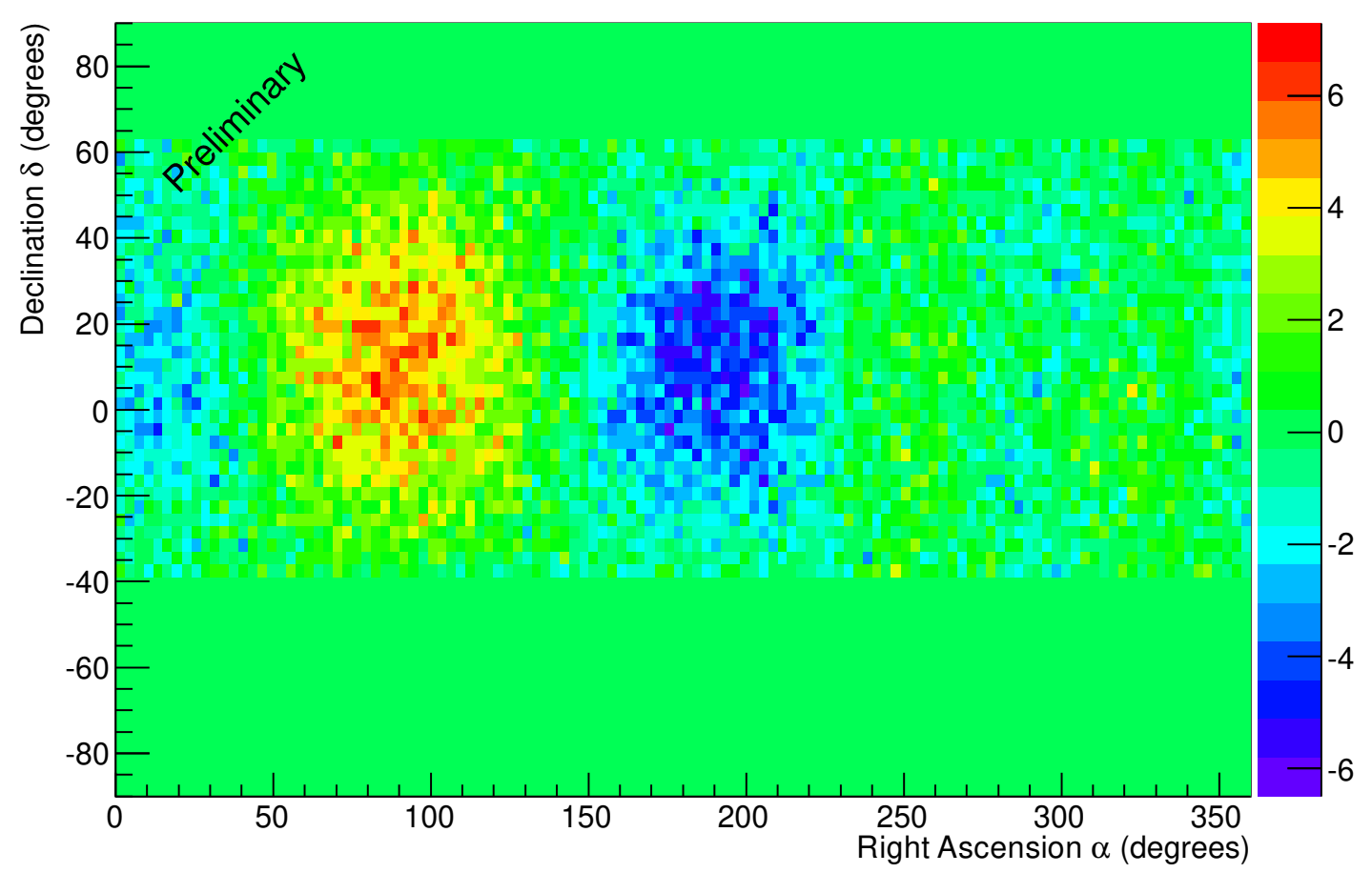

Figure 1: Statistical significance map of anisotropy measured by GRAPES-3

in southern hemisphere. The anisotropy shown in Fig.2 in three different declination bands shows the excess about $\alpha=90^{\circ}$ and deficit about $\alpha=190^{\circ}$. It is to be noted that the GRAPES- 3 is located at latitude of $11.4^{\circ} \mathrm{N}$ and beyond $\delta=+40^{\circ}$ and $\delta=-40^{\circ}$, the anisotropy is not significant due to poor statistics with the subset of the data used in this analysis.

\section{Summary}

Preliminary results of anisotropy measured with a subset of the GRAPES-3 data from 2000 to 2006 are presented in this paper. The results indicate that the GRAPES-3 could observe the anisotropy extending to both the hemispheres due to its near equatorial location. An on-going analysis effort with higher statistics and a better understanding of various systematics is expected to complement the observations from both the hemisphere experiments.

\subsection{Acknowledgments}

We thank D.B. Arjunan, G.P. Francis, V. Jeyakumar, S. Kingston, K. Manjunath, S. Murugapandian, S. Pandurangan, B. Rajesh, K. Ramadass, V. Santoshkumar, M.S. Shareef, C. Shobana, R. Sureshkumar and other colleagues for their help in running and maintenance of the GRAPES-3 experiment.

\section{References}

[1] Blasi P 2013 Astron. Astrophys. Rev. 2170 


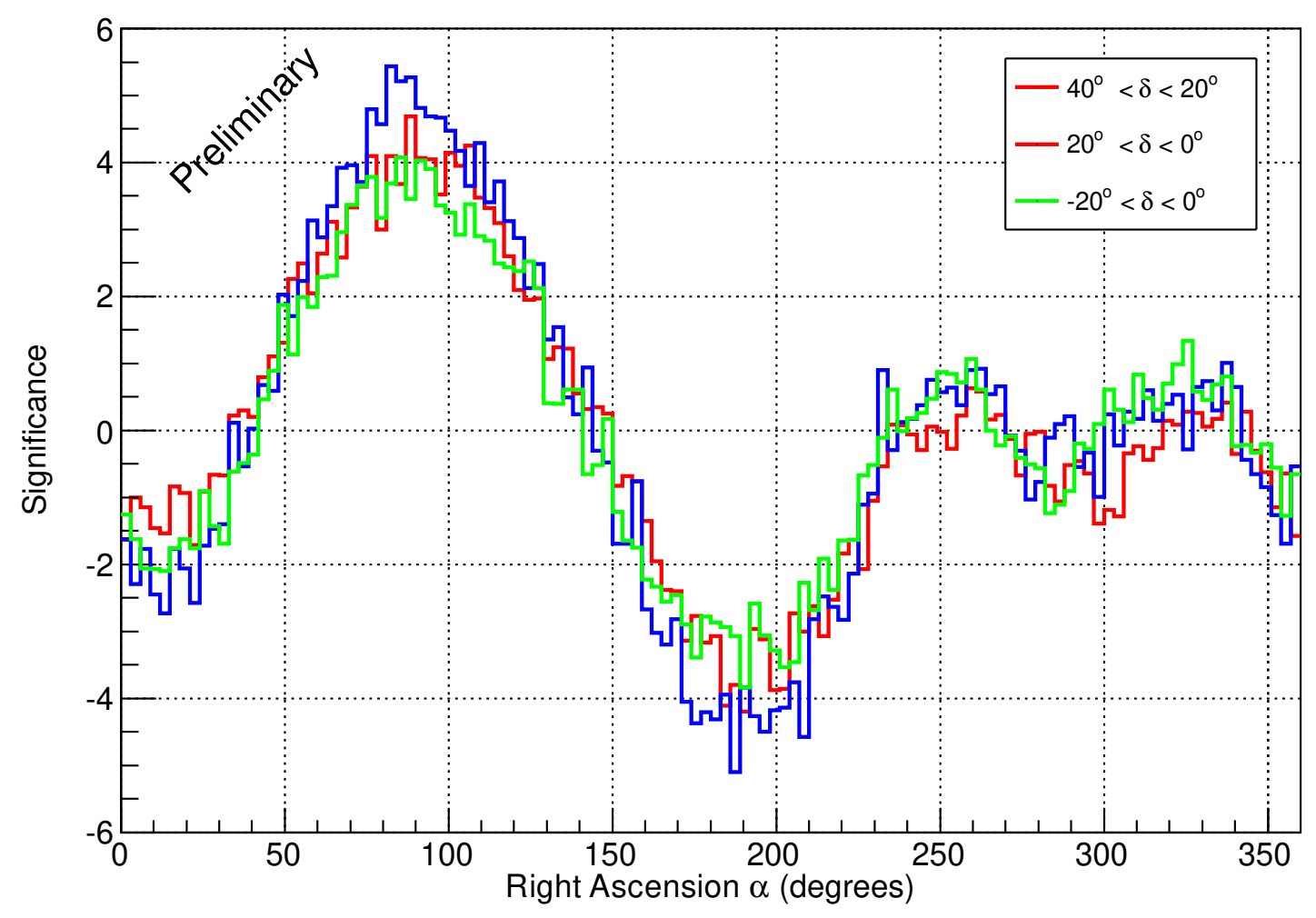

Figure 2: Statistical significance of anisotropy in different declination bands

[2] Sciascio G Di and Iuppa R arXiv:1407.2144

[3] Ahlers M 2016 Phys. Rev. Lett. 117151103

[4] Amenomori M et al. 2017 Astrophys. J. 836153

[5] Bartoli B et al. 2015 Astrophys. J. 80990

[6] Abdo A A et al. 2008 Phys. Rev. Lett. 101221101

[7] Abeysekara A U et al. 2018 Astrophys. J. 86557

[8] Aglietta M et al. 2009 Astrophys. J. Lett. 692 L130

[9] Aartsen M et al. 2013 Astrophys. J. 76555

[10] Aartsen M et al. 2016 Astrophys. J. 826220

[11] Gupta S K et al. 2005 Nucl. Instrum. Meth. A 540 311-23

[12] Mohanty P K et al. 2009 Astropart. Phys. 31 24-36

[13] Gupta S K et al. 2012 Expt. Astron. 35 507-26

[14] Hayashi Y et al. 2005 Nucl. Instrum. Meth. A 545 643-57

[15] Tanaka H et al. 2012 J. Phys. G: Nucl. Part. Phys. 39025201

[16] Oshima A et al. 2010 Astropart. Phys. 33 97-107

[17] LI T \& MA Y 1983 Astrophys. J. 272 317-324 